\section{Efficient algorithm for decoding Layered Space-Time Codes}

\author{
D. Wübben, R. Böhnke, J. Rinas, V. Kühn and \\ K.D. Kammeyer
}

Layered space-time codes have been designed to exploit the capacity advantage of multiple antenna systems in Rayleigh fading environments. In this paper, a new efficient decoding algorithm based on $\mathrm{QR}$ decomposition is presented. It needs only a fraction of computational effort compared to the standard decoding algorithm requiring the multiple calculation of the pseudo inverse of the channel matrix.

Introduction: In a Rayleigh fading environment multiple antenna systems provide an enormous increase in capacity compared to single antenna systems. To take advantage of multiple antennas space-time codes (STC) have been introduced to use space as a second dimension of coding. Layered space-time (LST) codes are a special kind of STC with the advantage of a feasible decoding complexity [1]. For these LST we introduce a new, efficient way of decoding based on the QR-decomposition.

System description: In this letter we consider a LST coding system as described in $[1,2]$ with $n_{T}$ transmit and $n_{R}$ receive antennas. Let $\mathbf{c}=\left(c_{1}, \ldots, c_{n_{T}}\right)^{T}$ denote the vector of transmitted symbols from a finite complex alphabet, then the corresponding receive vector $\mathbf{x}=\left(x_{1}, \ldots, x_{n_{R}}\right)^{T}$ is

$$
\mathbf{x}=\mathbf{H} \cdot \mathbf{c}+\boldsymbol{\nu} .
$$

where $\boldsymbol{\nu}=\left(\nu_{1}, \ldots, \nu_{n_{R}}\right)^{T}$ depicts white gaussian noise. The channel matrix

$$
\mathbf{H}=\left(\begin{array}{ccc}
h_{1,1} & \cdots & h_{1, n_{T}} \\
\vdots & \ddots & \vdots \\
h_{n_{R}, 1} & \cdots & h_{n_{R}, n_{T}}
\end{array}\right)
$$

contains the i.i.d. complex fading gains $h_{j, i}$ from transmit antenna $i$ to receive antenna $j$, which we assume to be constant for a frame length $L$ and perfectly known by the receiver.

Decoding LST: In [3] successive interference cancellation is used to decode distinct layers employing a nulling vector which is computed on basis of the Moore-Penrose pseudoinverse of a modified matrix of $\mathbf{H}$ in every iteration. To derive error bounds for LST codes another way of decoding was presented in [2] using the QR decomposition of the channel matrix $\mathbf{H}$. Thereby the $n_{R} \times n_{T}$ channel matrix $\mathbf{H}$ is factorized by $\mathbf{H}=\mathbf{Q} \cdot \mathbf{R}$ using the $n_{R} \times n_{T}$ unitary matrix $\mathbf{Q}$ and the $n_{T} \times n_{T}$ upper triangular matrix $\mathbf{R}$. Let $\mathbf{h}_{k}$ denote column $k$ of $\mathbf{H}$ and $\mathbf{q}_{k}$ denote the $k$ th column of $\mathbf{Q}$. Then the $\mathrm{QR}$ decomposition becomes:

$$
\left(\mathbf{h}_{1} \ldots \mathbf{h}_{n_{T}}\right)=\left(\mathbf{q}_{1} \ldots \mathbf{q}_{n_{T}}\right) \cdot\left(\begin{array}{ccc}
r_{1,1} & \cdots & r_{1, n_{T}} \\
& \ddots & \vdots \\
\mathbf{0} & & r_{n_{T}, n_{T}}
\end{array}\right) .
$$

By left multiplying equation (1) with the Hermitian matrix of $\mathbf{Q}$ a modified receive vector $\mathbf{y}$ is created:

$$
\mathbf{y}=\mathbf{Q}^{H} \cdot \mathbf{x}=\mathbf{R} \cdot \mathbf{c}+\boldsymbol{\eta} .
$$

Since $\mathbf{Q}$ is unitary, the statistical properties of the noise term $\boldsymbol{\eta}=\mathbf{Q}^{H} \cdot \boldsymbol{\nu}$ remain unchanged. Element $k$ of vector $\mathbf{y}$ becomes $y_{k}=r_{k, k} \cdot c_{k}+\eta_{k}+d_{k}$ and depends on the transmitted signal $c_{k}$, the noise $\eta_{k}$, and an interference term $d_{k}=\sum_{j=k+1}^{n_{T}} r_{k, j} \cdot c_{j}$. Since $\mathbf{R}$ is upper triangular, $d_{k}$ is independent of $c_{1}, \ldots, c_{k-1}$ and thus $c_{n_{T}}$ can be estimated immediately by $\hat{c}_{n_{T}}=\mathcal{Q}\left[y_{n_{T}} / r_{n_{T}, n_{T}}\right]$ employing the quantization function $\mathcal{Q}[\cdot]$ appropriate to the signal constellation. Furthermore, for $1 \leq k<n_{T}$ the interference term $d_{k}$ can be cancelled assuming that all previous decisions $\hat{c}_{k+1}, \ldots, \hat{c}_{n_{T}}$ are correct and $z_{k}=y_{k}-\hat{d}_{k}=r_{k, k} \cdot c_{k}+\eta_{k}$ may be used to determine $\hat{c}_{k}=\mathcal{Q}\left[z_{k} / r_{k, k}\right]$.

The order of detection is crucial for the error probability of the LST system [3] due to the risk of error propagation. The sequence of detection is achieved by permutating the rows of $\mathbf{c}$ and the corresponding columns of $\mathbf{H}$ and thereby results in different matrices $\mathbf{Q}$ and $\mathbf{R}$. The optimum $\mathbf{R}$ maximizes the SNR in each step of the detection process (equals maximizing $\left|r_{k, k}\right|$ for $k=n_{T}, \ldots, 1$ ) and can be found by performing $\mathcal{O}\left(n_{T}^{2} / 2\right)$ QR decompositions of permutations of $\mathbf{H}[4]$.

Sorted $Q R$ decomposition: We now introduce a much more efficient heuristic approach that comes close to the error performance of the global optimization. It is basically an extension of the modified Gram-Schmidt algorithm by ordering the columns of $\mathbf{H}$ in each orthogonalization step.

By applying Gram-Schmidt algorithm the QR decomposition computes the matrix $\mathbf{R}$ line by line from top to bottom and the matrix $\mathbf{Q}$ columnwise from left to right. For a given $\mathbf{H}=\left(\mathbf{h}_{1} \ldots, \mathbf{h}_{n_{T}}\right)$ it calculates $\mathbf{q}_{1}$ of unit length and $r_{1,1}=\left|\mathbf{h}_{1}\right|$ to fulfill $\mathbf{h}_{1}=r_{1,1} \cdot \mathbf{q}_{1}$. In the next step the components of $\mathbf{h}_{2}$ in the direction of $\mathbf{q}_{1}$ are cancelled and $\mathbf{q}_{2}$ of unit length and $r_{2,1}$ and $r_{2,2}$ are computed to fulfill $\mathbf{h}_{2}=r_{2,1} \cdot \mathbf{q}_{1}+r_{2,2} \cdot \mathbf{q}_{2}$. The computation of the next steps are analog, thus the diagonal elements $r_{k, k}$ are depicting the length of $\mathbf{h}_{k}$ orthogonal to $\mathbf{q}_{1}, \ldots, \mathbf{q}_{k-1}$ and $r_{k, 1}, \ldots, r_{k, k-1}$ describing the projection of $\mathbf{h}_{k}$ into the vector space spanned by $\mathbf{q}_{1}, \ldots, \mathbf{q}_{k-1}$. Consequently the diagonal elements are calculated from $r_{1,1}$ to $r_{n_{T}, n_{T}}$, but as observed earlier it would be optimal to maximize the 
$\left|r_{k, k}\right|$ in every decoding step, that means from $r_{n_{T}, n_{T}}$ to $r_{1,1}$

To maximize the diagonal elements from $n_{T}$ to 1 by applying only one QR decomposition the fundamental idea of our algorithm is to find the permutation of $\mathbf{H}$ that minimizes each $\left|r_{k, k}\right|$ with $k$ running from 1 to $n_{T}$, leaving all $r_{j, j}$ with $j<k$ unchanged. This minimizes the diagonal elements in every decomposition step and thereby intends maximal diagonal elements $\left|r_{i, i}\right|$ in the succeeding steps $i>k$. The only change to the modified Gram-Schmidt algorithm consists of a reordering of the columns of $\mathbf{H}$ according to their minimum length orthogonal to the vector space already spanned by $\mathbf{q}_{1}, \ldots, \mathbf{q}_{k-1}$. This is done in line (4) and (5) of Fig. 1, with the permutation vector $\mathbf{p}$ storing the used reordering of $\mathbf{H}$ and thereby the order of decoding.

Simulation Results: The error performance of the proposed sorted QR decomposition is compared to the unsorted QR decomposition and to the standard LST decoding algorithm (V-BLAST, [3]) for QPSK symbols. The bit error rates (BER) of the monte carlo simulations for a system with $n_{T}=8$ and $n_{R}=12$ antennas are shown in Fig. 2. The strong impact of ordering the QR decomposition is obvious and only a small difference of approximately $0.5 \mathrm{~dB}$ related to LST decoding for a BER of $10^{-5}$ is visible. For systems with less antennas, e.g. $n_{T}=4$ and $n_{R}=8$, this performance loss vanishes.

Summary and Conclusions: We have described a new decoding algorithm for LST codes. The algorithm bases on the Gram-Schmidt QR decomposition and requires less computational effort in comparison to the standard detection algorithm with only small degradation in error performance. Due to the efficiency of decoding the feasibility of such a system in the consumer market is enhanced.

\section{References}

[1] G. J. Foschini. Layered Space-Time Architecture for Wireless Communication in a Fading Environment when Using Multiple Antennas. Bell Labs Technical Journal, 1(2):41-59, Autumn 1996.

[2] D. Shiu and J.M. Kahn. Layered Space-Time Codes for Wireless Communications using Multiple Transmit Antennas. In IEEE Proceedings of International Conference on Communications (ICC'99), Vancouver, B.C., June 6-10 1999.

[3] P. W. Wolniansky, G. J. Foschini, G. D. Golden, and R. A. Valenzuela. V-BLAST: An Architecture for Realizing Very High Data Rates Over the Rich-Scattering Wireless Channel. In IEEE Pro- ceedings of ISSSE-98, Pisa, Italy, 29. September 1998.

[4] G. J. Foschini, G. D. Golden, A. Valenzela, and P. W. Wolniansky. Simplified Processing for High Spectral Efficiency Wireless Communications Emplying Multi-Element Arrays. IEEE Journal on Selectes Areas in Commununications, 17(11):1841-1852, November 1999.

\section{Author's affiliations:}

D. Wübben, R. Böhnke, J. Rinas, V. Kühn and K.D. Kammeyer (University of Bremen, Department of Communications Engineering, P.O. Box 3304 40, D-28334 Bremen, Germany, Fax: +(49)-421/2183341, E-mail: \{wuebben, boehnke, rinas, kuehn, kammeyer\}@comm.uni-bremen.de

\section{Figure 1}

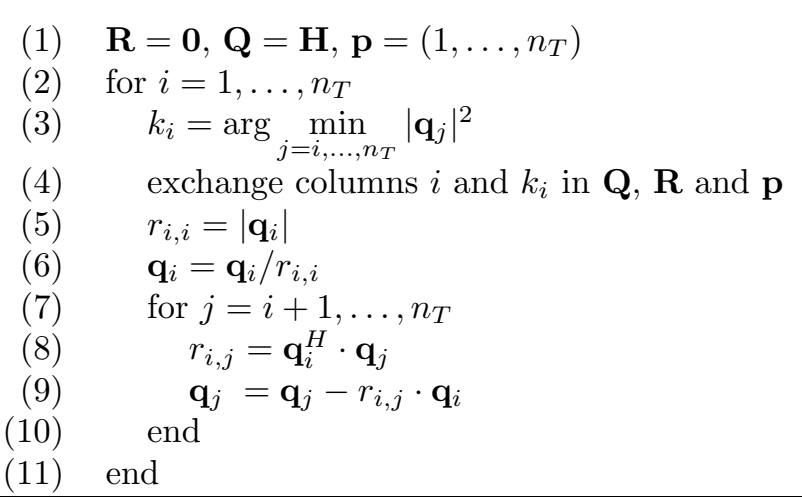

\section{Figure 2}

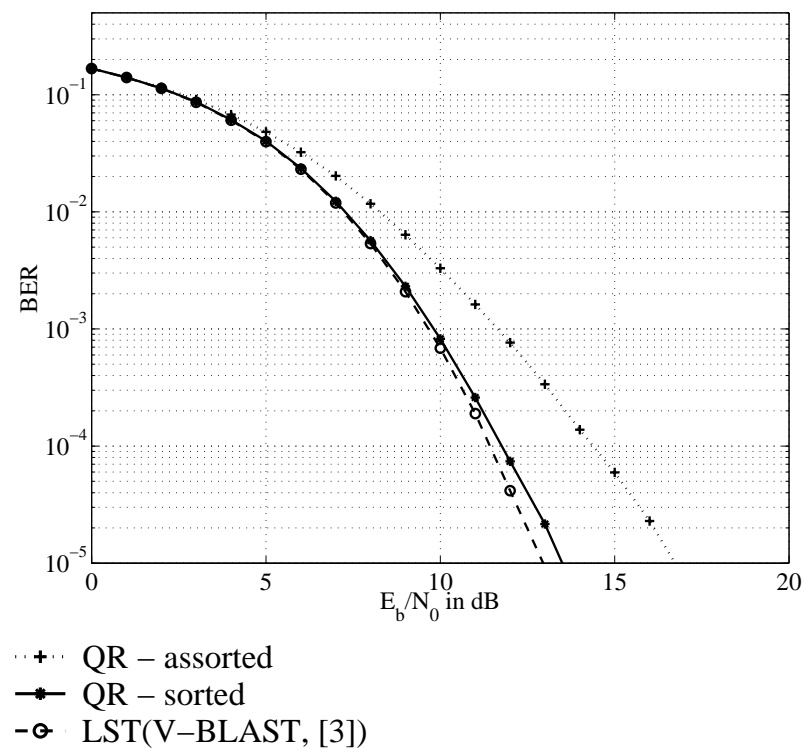

Simulation with $n_{T}=8$ and $n_{R}=12$ antennas where the transmitted signal power is normalized to $n_{R}$. 